\title{
Penile metastases originating from a pancreatic primary tumor: a case report
}

\author{
Tiffany L. Tello • Youssef H. Zeidan • Karl Bush • \\ Erich Schwartz • Pamela Kunz • Daniel T. Chang
}

Received: 16 October 2012 / Accepted: 25 October 2012 /Published online: 22 December 2012

(C) Springer-Verlag Berlin Heidelberg 2012

\begin{abstract}
Penile metastases are exceedingly rare. When they occur, they typically arise from a primary tumor in the genitourinary system. Here, we report a case of biopsyproven penile metastasis originating from a primary pancreatic adenocarcinoma. A 67-year-old man presented with a 5month history of progressive penile pain and difficulty urinating. Several penile corporeal nodules and plaques were present and a biopsy of a lesion was consistent with metastatic adenocarcinoma. Computed tomography of the abdomen revealed a $6.3-\mathrm{cm}$ infiltrative mass of the pancreatic body. Fine needle aspirate of the mass was consistent with pancreatic adenocarcinoma. The pathology specimens from the pancreatic mass and penile nodule were cytologically identical. The patient was offered palliative radiation to the penis for pain control. External beam radiation was carried out successfully to this challenging location with the
\end{abstract}

\section{T. L. Tello}

School of Medicine, University of Colorado Denver,

Denver, USA

Y. H. Zeidan $\cdot$ K. Bush • D. T. Chang

Department of Radiation Oncology,

Stanford University School of Medicine,

Stanford, USA

E. Schwartz

Department of Pathology, Stanford University School of Medicine,

Stanford, USA

P. Kunz

Department of Medicine, Division of Medical Oncology,

Stanford University School of Medicine,

Stanford, USA

D. T. Chang $(\bowtie)$

Department of Radiation Oncology,

Stanford University Medical Center,

875 Blake Wilbur Drive,

Stanford, CA 94305-5847, USA

e-mail: dtchang@stanford.edu use of a custom setup. Following radiation therapy, the patient reported a 70-80\% decrease in pain and nearly all of the clinically evident penile disease had cleared. To the best of our knowledge, this is the first case of penile metastases originating from a pancreatic adenocarcinoma reported in the USA and the second case ever reported worldwide.

Keywords Penile metastasis · Pancreatic cancer · External beam radiation therapy $\cdot$ Case report

\section{Introduction}

Penile metastases from malignancies are rare, with less than 250 cases reported in the literature [1]. Approximately $70 \%$ of the reported cases are from a genitourinary primary tumor, with colonic adenocarcinoma representing the next most common category $(22 \%)[1,2]$. Penile metastases from a pancreatic primary tumor are exceedingly rare, with only one case reported to our knowledge [3]. Herein, we report a case of biopsy-proven penile metastasis originating from a pancreatic primary tumor.

\section{Case presentation}

A 67-year-old Hispanic man presented to the emergency room with a 5-month history of penile pain that had progressed to include penile firmness and difficulty urinating. Initially, 5 months prior to admission, he began noticing an abnormal lesion on his penile shaft along with intermittent penile pain. After testing positive for herpes simplex virus I and II, he was initially treated with acyclovir without relief. Over the next several months, his symptoms worsened, including the development of pain radiating from his perineum to his penis and the appearance of several hard 
plaques on the penile shaft. A dermatologist performed a punch biopsy that revealed adenocarcinoma with lymphovascular invasion thought to be metastatic from a genitourinary or gastrointestinal primary (Fig. 1a).

He was scheduled to follow-up with urology, but presented to the emergency room prior his scheduled appointment due to increasing penile pain. Other symptoms included appetite loss and an unintentional weight loss of more than $9 \mathrm{~kg}$. A computed tomography (CT) of the abdomen and pelvis revealed a $6.3-\mathrm{cm}$ infiltrative mass involving the pancreatic body with extensive local lymphadenopathy and encasement of the hepatic, gastroduodenal, and proximal splenic arteries. The main portal vein, portal splenic confluence, and superior mesenteric vein were encased and narrowed (Fig. 2). Pelvic magnetic resonance imaging (MRI) showed metastatic disease to the penis, pelvic lymph nodes, adductor musculature, sacrum, and iliac bones (Fig. 3). His CA-19-9 at the time of admission was elevated to $45,180 \mathrm{U} / \mathrm{ml}$.

The patient subsequently underwent an upper endoscopic ultrasound that showed a large hypoechoic pancreatic neck and body mass. A fine needle aspirate (FNA) of the lesion was abundantly cellular with clusters and sheets of malignant cells. The cytologic features were consistent with pancreatic adenocarcinoma, with similar morphology to the initial penile biopsy (Fig. 1a-d).

The patient was referred to Radiation Oncology for consideration of palliative radiation therapy. Physical exam was remarkable for several penile corporeal nodules and plaques, a thickened penile shaft, and multiple ulcers along

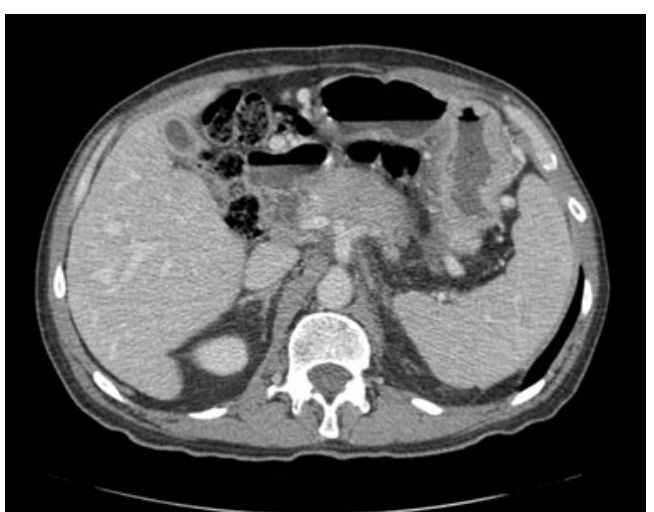

Fig. 2 Axial CT image showing $6.3 \mathrm{~cm}$ pancreatic body mass encasing the celiac artery and extensive local lymphadenopathy

the glans, particularly at the junction with the corpora. $\mathrm{He}$ had exquisite tenderness to palpation. There was significant bilateral inguinal lymphadenopathy.

The FNA of the pancreatic mass was compared with the initial penile biopsies and the lesions were morphologically indistinguishable. Although pancreatic adenocarcinoma has no diagnostic immunohistochemical staining profile, stains performed on the penile punch biopsy demonstrated that the adenocarcinoma strongly expressed CK7 and was focally positive for CK20. The pancreatic biopsy material showed an identical phenotypic expression profile (Fig. 1a-d). Overall, the findings were consistent with a pancreatic metastasis to the penis. The patient was offered palliative radiation for pain control.

A planning CT scan was performed with the patient lying supine. The penis was fixed pointing anteriorly with tape.
Fig. 1 Photomicrographs of surgical and FNA pathology specimens. (a) Hematoxylin and eosin (H\&E) stain of tissue from initial penile punch biopsy at $\times 40$. (b) H\&E stain of cytology from pancreatic mass FNA at $\times 40$. The H\&E tumor samples from both sites are arranged in an identical fused glandular pattern. (c) CK7 stain of tissue from initial penile punch biopsy at $\times 40$. (d) CK7 stain of cytology from pancreatic mass FNA at $\times 40$. Both tumor samples are strongly CK7 positive

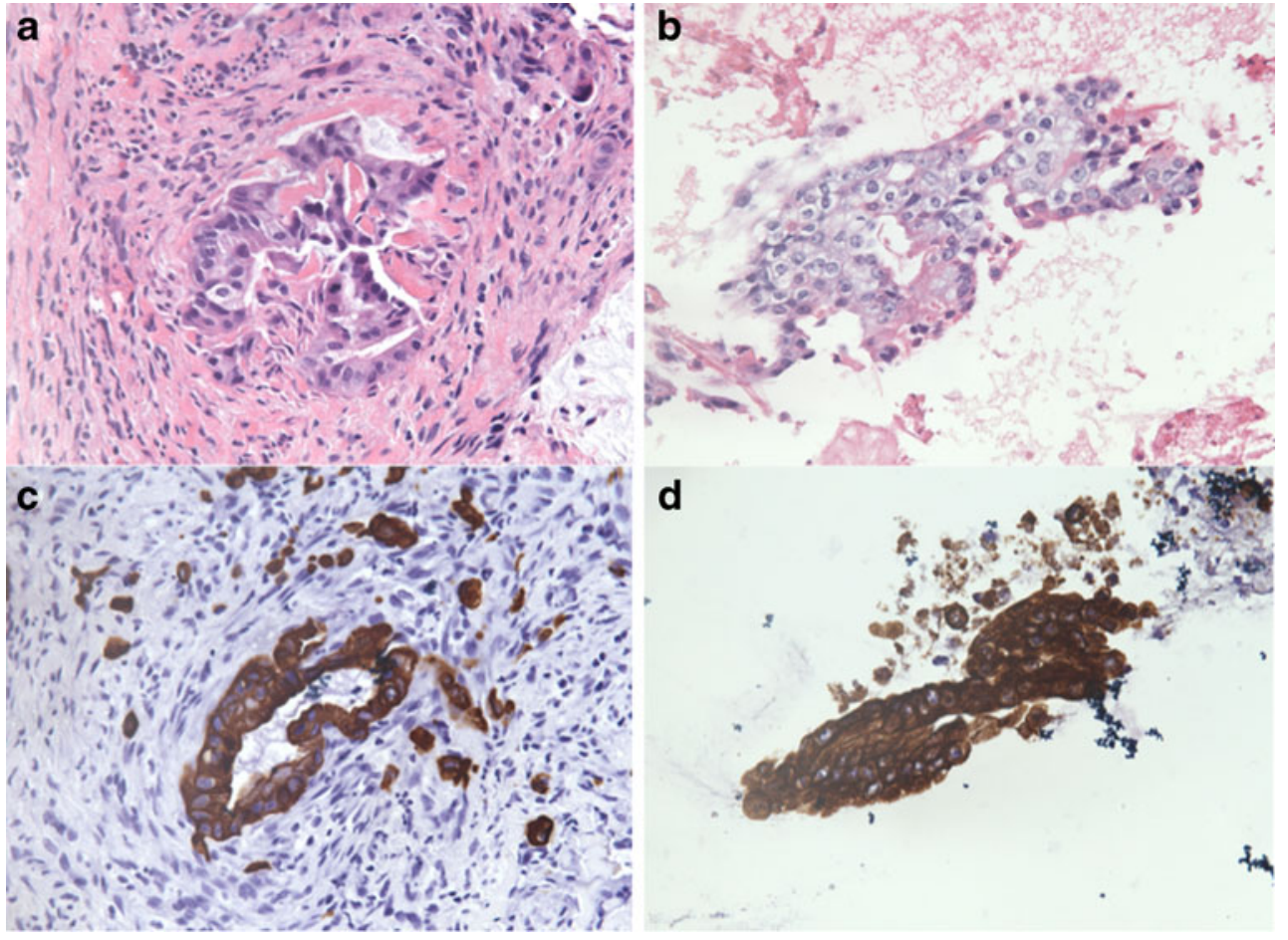


Fig. 3 MRI T1 post-

gadolinium axial images showing adductor muscle masses (a) and (b) and penile metastases (c) and (d)

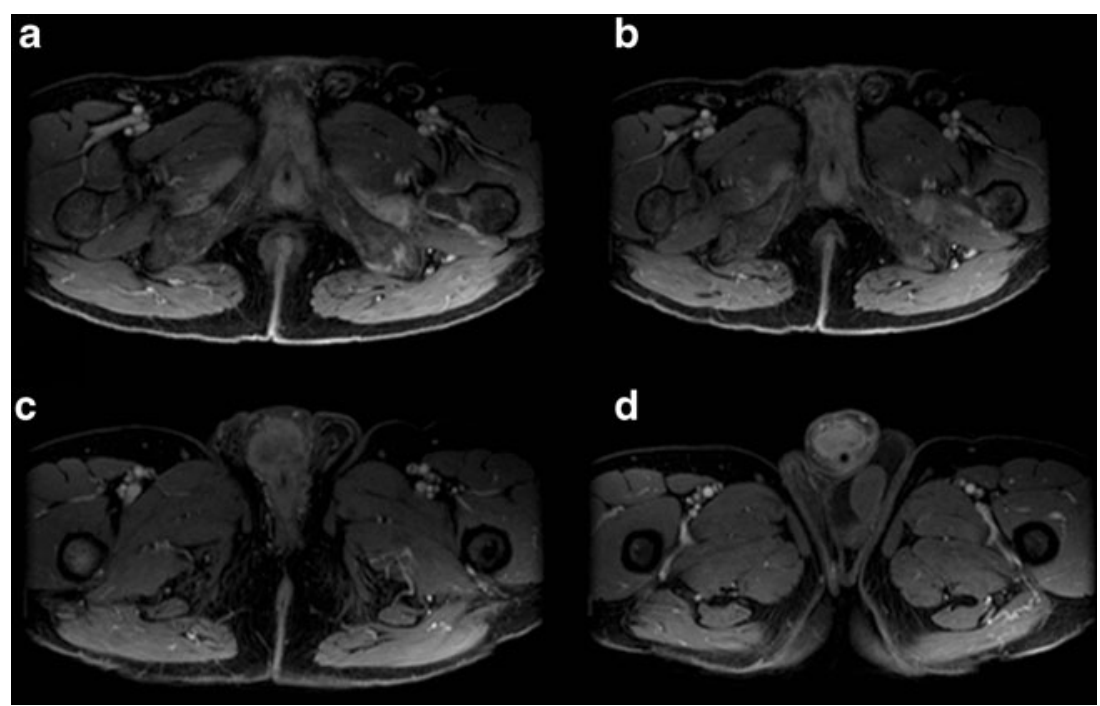

Because the lesion involved the surface of the skin, a 1-cmthick custom cylindrical wax bolus was used initially to ensure that the surface of the skin received adequate dose (Figs. 4a, b and 5a, b). The target volume included the entire penis down the penile bulb. An anterior and opposing posterior field technique was chosen.

After day 2 of treatment, the patient began complaining of groin pain, and review of his CT scan shows metastatic involvement of the bilateral adductor hip muscles. In order to treat this, the radiation beams needed to be widened to include this area. We decided at this point to create large wax blocks on day 3 of radiation in order to regulate the radiation source-to-skin distance of the anterior directed beam to produce a more even dose across the penis (Figs. 4c and 5c). The thickness of the patient was also made more uniform across the treatment fields, which reduced the lateral groin dose (see Fig. 4b, c). The wax blocks, shaped like a clamp, were larger and also allowed for improving the stabilization of the penis in an upright position. A stack of folded sheets was secured inferiorly between the leg immobilizer and the wax blocks in order to prevent sliding of the latter (Fig. 5c). Finally, the light field of the anterior beam was used to verify the proper positioning of the penis prior to each fraction. We used $6 \mathrm{MV} / 15 \mathrm{MV}$ photon beams delivering a total of $30 \mathrm{~Gy}$ in ten treatment fractions over 2 weeks. A sample of the crosssectional view of the plan is shown in Fig. 4c where the PTV is shown in red and the $95 \%$ isodose line in green. The Foley catheter was a reliable setup guide on the $\mathrm{kV}$ images, taken twice weekly. Furthermore, to confirm that the appropriate dose was being delivered to the surface of the penis, thermoluminescent dosimeters (TLDs) were placed on the penile shaft (Fig. 5d). The prescribed dose was 300 cGy per treatment. The readings of the TLDs were 363 cGy (121\% of prescribed dose), 360 cGy (120\% of prescribed dose), and 314 cGy (105\% of prescribed dose).
The patient reported a $50 \%$ decline in penile pain following completion of radiation therapy. Acute side effects included grade II dermatitis and significant swelling of the penile shaft that occurred after the first five treatments. At that point, the wax bolus was discontinued in order to decrease the radiation dose to the skin. He was also treated with a steroid taper that improved the swelling significantly. Systemic chemotherapy (Gemzar, $1,000 \mathrm{mg} / \mathrm{m}^{2}$ weekly) was initiated on day 4 of radiation.

Three months later, on physical exam, the patient's penis had responded approximately $90 \%$ in size (Fig. 6). The penis and glans was soft throughout the glans and shaft with only a 1-2-cm firm residual tumor at the ventral surface along the urethra. By then, patient had developed urethral stricture requiring a suprapubic catheter. He reported however that his pain had decreased by $70-80 \%$.

\section{Discussion}

To the best of our knowledge, this is the first case of penile metastases originating from a pancreatic adenocarcinoma reported in the USA and the second case ever reported worldwide [3]. The most common locations of pancreatic cancer metastasis are the liver, regional lymph nodes, and peritoneal cavity, followed closely by the lung [4]. Less common sites of metastatic pancreatic cancer include the brain, bone, and adrenal glands [4]. Case reports have described unusual sites of metastatic disease such as the skin, skeletal muscle, myocardium, stomach, appendix, kidney, and prostate [5-12]. Pancreatic cancer is known to metastasize by a variety of mechanisms. Most commonly, it spreads hematogenously through the portal venous system to the liver [13]. It can also directly invade surrounding visceral organs or can metastasize to any surface within the peritoneal cavity by seeding. It spreads to the lungs hematogenously by tumor thrombi and can also move via the lymphatic system through permeation, 
Fig. 4 RT planning. Two fields (AP/PA) were used to treat the target volume. Dose

distributions from the fields are shown for the cases of (a) no bolus, (b) $1 \mathrm{~cm}$ bolus, and (c) custom wax bolus. The target volume (PTV) is shown in red and $95 \%$ isodose line is shown in green (30 Gy)

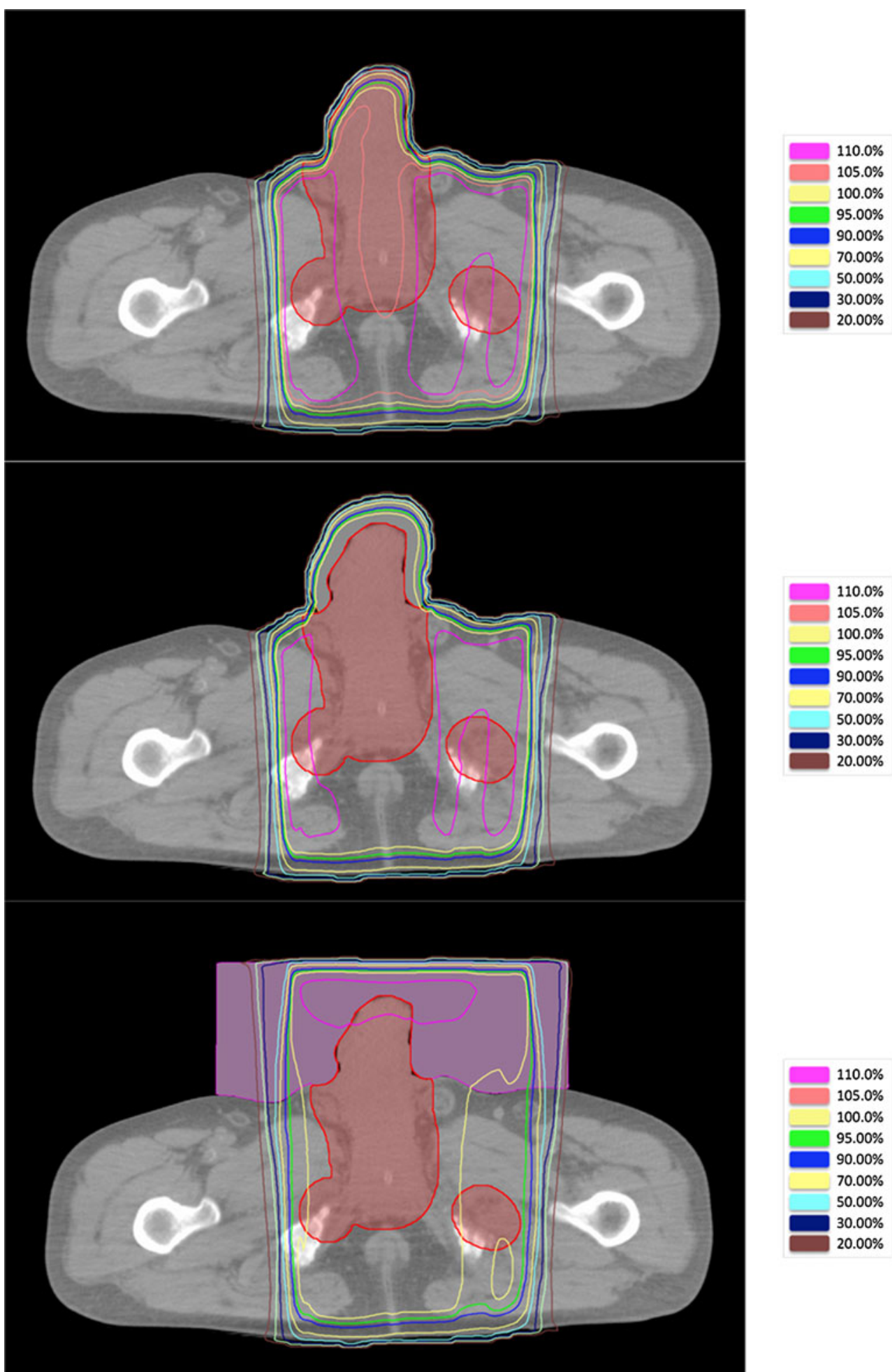

embolization, and retrograde spread due to lymphatic obstruction within the pancreas $[4,13]$. Finally, there is the chemotaxis theory, by which cancer cells with high expression of specific chemokine receptors may spread to specific sites that express high levels of the corresponding ligand [6, 14]. In this case, our patient had diffuse involvement of the inguinal and pelvic lymph nodes, peritoneal and pelvic vasculature, and adjacent musculoskeletal masses. Therefore, any of these mechanisms could be responsible for the presence of penile metastasis in our patient.

Cases of penile metastasis from genitourinary and gastrointestinal primaries have been previously discussed in the 
Fig. 5 Setup with (a) $1 \mathrm{~cm}$ custom wax bolus, (b) styrofoam block, and (c) custom wax block. (d) Thermoluminescent dosimeters (TLDS) were placed on the skin of the penile shaft on the first day of treatment to measure skin dose

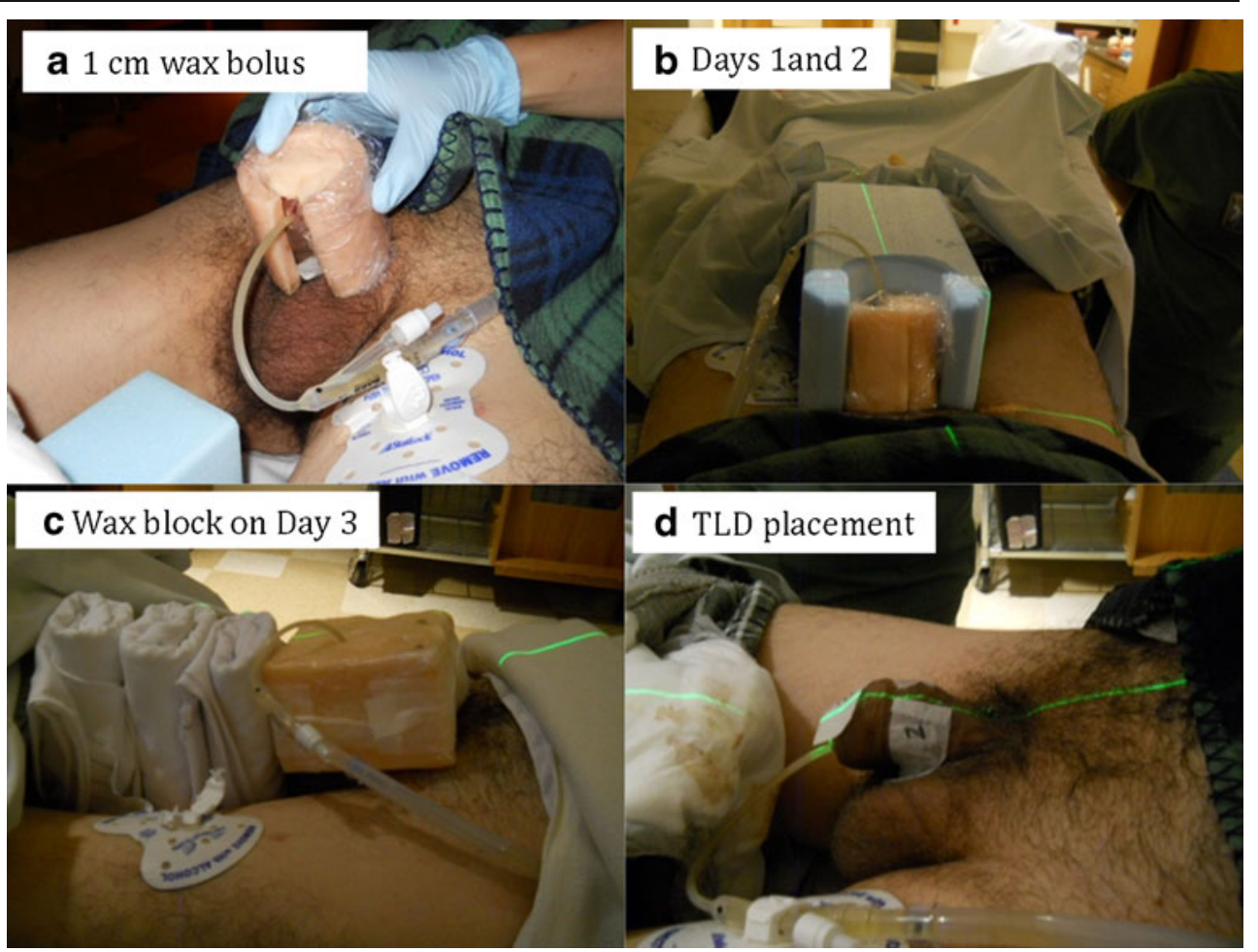

literature. Most commonly described are metastases from prostate and bladder carcinomas, with more than 200 cases reported $[15,16]$. Palliative radiation therapy was given in $15 \%$ of those cases. Nearby gastrointestinal sites such as the rectosigmoid colon account for $13 \%$ to of penile metastasis with fewer than 60 cases described [17]. Regardless of the primary site, treatment options include radical or partial penectomy, local excision, hormonal or chemotherapy, and radiation therapy (teletherapy or brachytherapy). Appropriate treatment selection should be personalized according to extent of disease, symptoms, and patient preference.

The penis presents several challenges with regards to radiation treatment. First, we had to create a reliable setup for precise delivery of radiation, while avoiding excess discomfort and pain for the patient. We ultimately designed a custom wax block with a thickness that matched the entire length of the penile shaft, which was fit into a central channel to cover it entirely. This wax block served several purposes: (1) to ensure adequate dose build-up along the surface of the shaft, (2) to maintain an upright position and minimize motion during treatment, and (3) to allow improved dose homogeneity to the lateral groins. Because of the irregular shape of the penis, the source-to-skin distance was not uniform along the length of the field and the thickness of the patient was also widely varying which lead to significant dose heterogeneity in the treated volume. By using tissue-equivalent wax blocks to match to the tip of the penis, the effects from these geometrical challenges were reduced, leading to a more uniform dose and an avoidance of unnecessarily high dose levels to the treated volume.
Light fields and $\mathrm{kV}$ images were used to verify setup and TLDs were used to estimate skin surface dose, as described in further detail above. Other techniques to treat the penis have been described, such as a water bath with the patient in a prone position or a plastic box with a hole in the middle [18]. In addition to EBRT, brachytherapy using molds and interstitial implants have been used for treating penile lesions [19, 20]. The anterior/posterior beam arrangement in this case was chosen in this case for two reasons. First, the need to cover

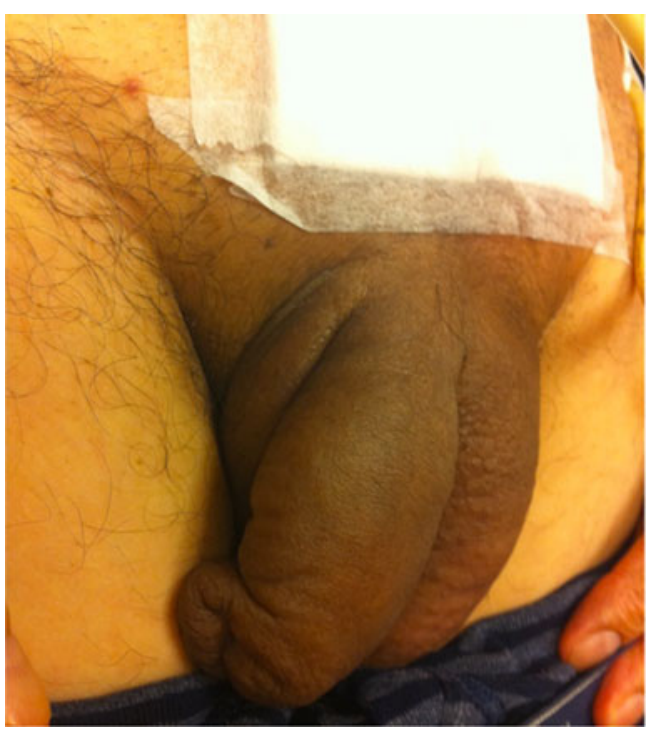

Fig. 6 Clinical response 3 months post-palliative radiation. Treatment area shows reduction of penile shaft metastatic burden and skin hyperpigmentation 
the deeper tissue including the penile bulb and prostate as well as the hip adductor muscles prevent simple cross-fire techniques that could be used to treat just the penile shaft. Secondly, due to the variability of the penis position, an anterior field would allow daily verification with the use of the anterior beam light field. Overall, we found this technique to be reproducible and tolerable for the patient.

In summary, this case report describes a rare presentation of pancreatic cancer with penile metastases manifesting as penile pain and urinary problems. This case presented a challenging location to palliate; however, this was performed successfully with the use of external beam radiation.

Acknowledgments The authors would like to thank the radiation therapy staff of LA-10 for assistance with taking images and Mr. Fred Van Den Haak for custom blocks design.

Conflict of interest The authors declare that they have no conflicts of interest.

\section{References}

1. Powell BL, Craig JB, Muss HB (1985) Secondary malignancies of the penis and epididymis: a case report and review of the literature. $\mathrm{J}$ Clin Oncol 3(1):110-116

2. Harper M, Arya M, Shah PJ (2002) A lump in the penis. J R Soc Med 95(1):38-39

3. Ahn TY, Choi EH, Kim KS (1997) Secondary penile carcinoma originated from pancreas. J Korean Med Sci 12(1):67-69

4. Blastik M, Plavecz E, Zalatnai A (2011) Pancreatic carcinomas in a 60-year, institute-based autopsy material with special emphasis of metastatic pattern. Pancreas 40(3):478-480

5. Borad MJ et al (2009) Skeletal metastases in pancreatic cancer: a retrospective study and review of the literature. Yale J Biol Med 82(1):1-6

6. Saif MW et al (2011) Cutaneous metastasis in a patient with pancreatic cancer. JOP 12(3):306-308
7. Val-Bernal JF et al (2000) Skin metastases as the presenting sign of pancreatic ductal adenocarcinoma in a renal transplant patient. Ann Saudi Med 20(3-4):253-256

8. Martino L et al (2004) Renal metastasis from pancreatic adenocarcinoma. Arch Ital Urol Androl 76(1):37-39

9. Filik L et al (2003) Appendicular metastasis from pancreatic adenocarcinoma. Int J Gastrointest Cancer 34(1):55-58

10. Merseburger AS et al (2005) A rare case of isolated prostate metastasis from primary pancreatic cancer. Urologe A 44(5):527-529

11. Robinson BW, Lewis RR (1982) Myocardial metastasis from carcinoma of pancreas presenting as acute myocardial infarction. J R Soc Med 75(7):560-562

12. Takamori $\mathrm{H}$ et al (2005) Metastatic gastric tumor secondary to pancreatic adenocarcinoma. J Gastroenterol 40(2):209-212

13. Kamisawa $\mathrm{T}$ et al (1995) Hematogenous metastases of pancreatic ductal carcinoma. Pancreas 11(4):345-349

14. Wang Z, Ma Q (2007) Beta-catenin is a promising key factor in the SDF-1/CXCR4 axis on metastasis of pancreatic cancer. Med Hypotheses 69(4):816-820

15. Philip J, Mathew J (2003) Penile metastasis of prostatic adenocarcinoma: report of two cases and review of literature. World J Surg Oncol 1(1):16

16. Senkul $\mathrm{T}$ et al (2002) Penile metastasis of prostatic adenocarcinoma. Int J Urol 9(10):597-598

17. Park JC et al (2009) Priapism secondary to penile metastasis of rectal cancer. World J Gastroenterol 15(33):4209-4211

18. Perez CA, Brady LW, Halperin EC, Schmidt-Ullrich RK (2004) Principles and practice of radiation oncology. In: Perez CA (ed) Brady LW. Lippincott, Philadelphia

19. Gerbaulet A, Lambin P (1992) Radiation therapy of cancer of the penis. Indications, advantages, and pitfalls. Urol Clin N Am 19 (2):325-332

20. Crook J et al (2002) Interstitial brachytherapy for penile cancer: an alternative to amputation. J Urol 167(2 Pt 1):506-511

\section{Consent}

Written and verbal informed consent was obtained from the patient for publication of this case report and any accompanying images. 